\title{
Identificação de bergenina e carotenóides no fruto de uchi (Endopleura uchi, Humiriaceae)
}

\author{
Lyege Amazonas M. MAGALHĀES ${ }^{1}$, Maria da Paz LIMA², Helyde Albuquerque MARINHO³, \\ Antonio Gilberto FERREIRA ${ }^{4}$ \\ RESUMO \\ O extrato obtido em metanol da polpa de frutos Endopleura uchi foi submetido a fracionamento utilizando-se técnicas \\ cromatográficas convencionais levando ao isolamento de bergenina. $\mathrm{Na}$ análise da composição dos carotenóides foi evidenciada \\ a predominância de $\beta$-caroteno $\left(16,57 \mu \mathrm{g}\right.$. $\left.\mathrm{g}^{-1}\right)$. Os isômeros do $\beta$-caroteno foram detectados, trans- $\beta$-caroteno $(89,3 \%)$, 13-cis- \\ $\beta$-caroteno (8\%) e 9-cis- $\beta$-caroteno (3\%). Considerando a importância do papel nutricional dos carotenóides e a bioatividade \\ do glicosídeo bergenina, esse estudo sugeriu o potencial desse fruto como alimento funcional.
}

PALAVRAS-CHAVE

Endopleura uchi, Bergenina, $\beta$-Caroteno.

\section{Identification of bergenin and carotenoids in the uchi (Endopleura uchi, Humiriaceae) fruit}

\section{ABSTRACT}

The extract obtained in methanol from the fruits pulp of Endopleura uchi was fractionated using conventional chromatographic tecniques to isolate bergenin. In the analysis of the composition of carotenoids was evidenced the predominance of $\beta$-carotene $\left(16.57 \mu \mathrm{g} \cdot \mathrm{g}^{-1}\right)$. The isomers of $\beta$-carotene: trans- $\beta$-carotene $(89.3 \%), 13$-cis- $\beta$-carotene $(8 \%)$ and 9 -cis- $\beta$-carotene $(3 \%)$ were detected. Considering the importance of the nutritional role of carotenoids and the bioactivity of the bergenina, this study suggested the potential of this fruit as functional food.

\section{KEYWORDS}

Endopleura uchi, Bergenin, $\beta$-Carotene.

\footnotetext{
1 Programa de Iniciação Científica, Instituto Nacional de Pesquisas da Amazônia. e-mail: Iyege@inpa.gov.br.

2 Instituto Nacional de Pesquisas da Amazônia, Coordenação de Pesquisas em Produtos Naturais - CPPN. e-mail: mdapaz@inpa.gov.br. Endereço de correspondência

3 Instituto Nacional de Pesquisas da Amazônia, Coordenação de Pesquisas em Ciências da Saúde - CPCS. e-mail: marinho@inpa.gov.br.

${ }^{4}$ Universidade Federal de São Carlos, Departamento de Química, Caixa Postal 676. e-mail: giba@dq.ufscar.br
} 


\section{INTRODUÇÃO}

Endopleura uchi (Huber) Cuatrec. [sin. Sacoglottis uchi Huber] (Humiriaceae) é uma espécie originária da Amazônia brasileira, encontrada em estado silvestre em mata de terra firme, dispersa por toda a Bacia Amazônica. A planta é conhecida na região como uchi, uxi ou uchi-pucu (Cuatrecasas, 1961; Schultes, 1979).

A madeira dessa espécie é utilizada na construção civil e naval, marcenaria e carpintaria. A casca da árvore é utilizada na forma de maceração para o tratamento de artrite, colesterol, diabete e como antiinflamatório (Corrêa, 1984).

O fruto é uma dupla oblongo-elipsóide de 5 a $7 \mathrm{~cm}$ de comprimento, 3 a $4 \mathrm{~cm}$ de diâmetro, com peso entre 50 e $70 \mathrm{~g}$ e apresenta coloração verde-amarelada ou parda-escuro quando maduro. É considerado um alimento de grande importância para subsistência de muitas comunidades rurais mais distantes, no entanto na última década tem sido detectado um mercado em expansão na área periurbana de Belém durante os quatro meses de frutificação, gerando renda para várias famílias. A polpa in natura é consumida pura bem como na fabricação de sorvete e licor. O fruto é também apreciado por vários animais silvestres, tornando-o favorito aos caçadores para construção de armadilhas de caça (Shanley, Luz \& Swingland, 2002).

Estudos fitoquímicos efetuados com essa espécie descrevem a ocorrência das isocumarinas bergenina e 8,10dimetoxibergenina e dos triterpenóides pentacíclicos, ácido masílinico e o seu éster masilinato de metila, no caule de um espécime coletado na Pará (Luna, Bento \& Sant`Ana, 2001; Luna et al., 2000). Os estudos com polpa de frutos de uchi o indicaram como fonte de ácidos graxos, fibras, esteróides, sais minerais, vitaminas $\mathrm{C}$ e E. Os principais ácidos graxos identificados foram o ácido oléico $(7,38 \%)$ e ácido palmítico $(3,78 \%)$. A composição do aroma nos frutos também foi avaliada, sendo identificados 42 componentes com predominância de 3,3-dimetil-2-butanol (18,8\%) e eugenol (14,0\%) (Marx et al., 2002).

Considerando a importância econômica e alimentar dos frutos de uchi para região amazônica, esse trabalho objetivou a descoberta de possíveis constituintes responsáveis pelas propriedades terapêuticas e/ou nutricional.

\section{MATERIAIS E MÉTODOS}

\section{PROCEDIMENTOS EXPERIMENTAIS GERAIS}

Os espectros de RMN foram obtidos em aparelho Bruker DRX-400 ( $\left.{ }^{1} \mathrm{H}: 400 \mathrm{MHz} ;{ }^{13} \mathrm{C}: 100 \mathrm{MHz}\right)$, utilizando DMSO$\mathrm{d} 6$ como solvente. $\mathrm{O}$ espectro de absorção na região do IV foi obtido em aparelho Perkim Elmer (Spectrum-2000), utilizando pastilha de $\mathrm{KBr}$. O ponto de fusão foi determinado no aparelho da Marconi (MA-381). Para o fracionamento do extrato obtido em metanol, foram utilizadas colunas cromatográficas em gel de sílica (Merck, 70-230 mesh) e celulose microcristalina Avicel (Merck). As placas cromatográficas foram reveladas com luz UV e solução alcoólica de vanilina/ácido sulfúrico. Para identificação dos carotenóides utilizou-se espectrofotômetro UV/Visível, Perkin-Elmer 552A, duplo feixe, com registrador acoplado.

\section{EXTRAÇÃO, FRACIONAMENTO E ISOLAMENTO DA SUBSTÂNCIA 1}

Os frutos maduros de Endopleura uchi foram coletados em dezembro de 2003 no Campus I do INPA em indivíduo previamente marcado (árvore ${ }^{0} 176$ ) e identificado durante a execução do projeto INPA/ODA (Ferreira \& Ramos, 1993). A polpa foi macerada à frio com hexano, metanol e metanol:água (1:1), sendo o extrato obtido em metanol $(16,56 \mathrm{~g})$ submetido à partição em diclorometano:água $(7: 3)$, seguida por acetato de etila. As fases orgânicas reunidas com base em cromatografia em camada delgada (CCD), foram fracionadas em coluna de sílica gel (70-230 mesh), eluídas em gradiente de polaridade [hexano:diclorometano (1:1); diclorometano; diclorometano:acetona $(95: 5) \rightarrow(1: 1)$; acetona e metanol], fornecendo 18 fraçōes. As frações 12 a 14 (1,46 g) eluídas em diclorometano:acetona (1:1) foram reunidas e refracionadas em coluna de celulose, eluída em gradiente [hexano; hexano:acetona $(9: 1) \rightarrow(1: 1)$; acetona e etanol] fornecendo 28 subfrações. As subfraçôes 23 e 24, eluídas em acetona, forneceram a substância $\mathbf{1}(269 \mathrm{mg})$, a qual foi purificada após várias recristalizaçōes em acetona.

\section{DETERMINAÇÃO DOS CAROTENÓIDES}

A determinação do teor de carotenóides na polpa do uchi, baseou-se no método preconizado por RodriguezAmaya (1977). Os pigmentos foram extraídos com acetona utilizando-se um homogeneizador, tipo liquidificador (aço inox), seguindo-se uma filtração a vácuo em funil de Büchner, várias vezes até descoloração total da polpa. O extrato obtido em acetona (contendo pigmento amarelo) foi submetido à partição em éter de petróleo:água. Para análise preliminar do número de carotenóides nas amostras, a fase etérea foi avaliada em cromatografia em camada delgada, utilizando-se como fase móvel, éter etílico:éter de petróleo (1:9).

Para hidrólise dos ésteres, a fase etérea foi submetida à saponificação com uma solução metanólica de $\mathrm{KOH}$ a $10 \%$ durante 12 horas, em repouso a temperatura ambiente. $\mathrm{Na}$ separação dos pigmentos utilizou-se coluna cromatográfica em MgO:Hiflosupercel (1:2) cujas fases móveis empregadas foram éter de petróleo e gradiente de éter de petróleo:acetona. As fraçôes obtidas foram concentradas em evaporador rotativo $\left(\mathrm{a} 35^{\circ} \mathrm{C}\right)$ até um volume suficiente para leitura em espectrofotômetro, registrado de 350 a $550 \mathrm{~nm}$. Para separação dos isômeros cis do $\beta$-caroteno foi utilizada a cromatografia em coluna de óxido de magnésio (Rodriguez-Amaya et al., 1976). 
$\mathrm{Na}$ identificação dos carotenóides considerou-se a ordem de eluição na coluna cromatográfica, espectro de absorção na região do UV/visível, valores de Rfem cromatografia em camada delgada comparativa e reação de isomerização. Verificou-se a isomerização transferindo-se a fração de $\beta$-caroteno para uma cubeta do espectrofotômetro e adicionando-se duas gotas de solução de iodo em éter de petróleo para a realização da leitura. Em seguida, essa fração foi exposta à luz e novamente a leitura foi registrada. $\mathrm{O}$ deslocamento para comprimento de onda menor é indicativo da presença de isômeros todotrans e para maior ou sem deslocamento indica a presença de isômeros cis.

Foi aplicada a Lei de Beer para quantificação dos carotenos em cada fração, de acordo com suas absorbâncias máximas e também a tabela de absortividades (Davies, 1976). O teor de vitamina $\mathrm{A}$ foi calculado com base na atividade de pró-vitamina A de cada carotenóide, segundo Bauerfeind (1972).

As análises foram efetuadas em duplicata e os experimentos em todas as etapas foram realizados ao abrigo da luz.

\section{RESULTADOS E DISCUSSÃO}

\section{IDENTIFICAÇÃO DE BERGENINA}

A estrutura da isocumarina bergenina (1) [sólido cristalino, p.f. $149,8^{\circ} \mathrm{C}$ ] foi definida com base nas análises dos espectros de IV, RMN de ${ }^{1} \mathrm{H}$ e ${ }^{13} \mathrm{C}$ e por comparação com dados da literatura (Seo et al., 1999). As principais bandas de absorção observadas no IV (KBr) foram em 3400 e $3291 \mathrm{~cm}^{-1}(\mathrm{OH})$, $1706 \mathrm{~cm}^{-1}(\mathrm{C}=\mathrm{O}), \quad 1609,1587$ e $1493 \mathrm{~cm}^{-1}(\mathrm{C}=\mathrm{C}$ de aromático).

$\mathrm{O}$ espectro de $\mathrm{RMN}$ de ${ }^{1} \mathrm{H}$ (Tabela 1) mostrou sinais característicos da bergenina: um sinal de aromático em $\delta$ 6,98 e de metoxila em $\delta 3,77(3 \mathrm{H}, s)$ além dos sinais típicos do C- glicosídeo. No espectro de RMN ${ }^{13} \mathrm{C}$ (Tabela 1), o sinal de carbonila foi verificado em $\delta 163,63$, a metoxila em $\delta 60,05$ e o grupo hidroximetileno em $\delta 61,33$.

O espectro de COSY $1 \mathrm{H}-1 \mathrm{H}\left(400 \mathrm{MHz}, \mathrm{DMSO}-\mathrm{d}_{6}\right)$, revelou o acoplamento dos hidrogênios metínicos em $\delta$ 4,98 (H-9) com $\delta 3,99(\mathrm{H}-14)$ e desse último com o hidrogênio oximetínico em $\delta 3,65(\mathrm{H}-13)$. Outros acoplamentos observados foram: $\delta 3,85(\mathrm{H}-16 \mathrm{a})-\delta 3,44(\mathrm{H}-16 \mathrm{~b}) ; \delta 3,65$ $(\mathrm{H}-13)-\delta 3,20$ (H-12); $\delta 3,57$ (H-11)- $\delta 3,20$ (H-12).

Os dados dos espectros de $\mathrm{RMN}$ de ${ }^{1} \mathrm{H}$ e ${ }^{13} \mathrm{C}$ foram similares aos descritos na literatura (Seo et al., 1999).

A substância 1, descrita nesse trabalho, foi isolada anteriormente a partir do caule de um espécime coletado no Pará (Luna et al, 2000) e tem sido relatada nesta família, na espécie Humiria balsamifera por sua atividade antiinflamatória (Harborne \& Baxter, 1993) bem como em casca da espécie africana Sacoglottis gabonensis (Ogan, 1971).<smiles>COc1c(O)cc2c(c1O)[C@H]1O[C@H](CO)[C@@H](O)[C@H](O)[C@H]1OC2=O</smiles>

Bergenina (1)

Outros registros de ocorrência desse C-glicosídeo são nos frutos de Ardisia colorata (Myrsinaceae) e folhas de Saxifraga stolonifera e Rodgersia podophylla (Saxifragaceae) (Taneyana et al., 1983; Chin \& Kim, 2006). Em experimentos com animais de laboratório, a bergenina tem mostrado potente ação hepatoprotetora (Lim et al., 2001; Lim et al., 2000; Lim et al., 2001).

\section{IDENTIFICAÇÃO DOS CAROTENÓIDES}

Foram identificados carotenóides com atividades de pró-vitamina A: $\beta$-caroteno e $\alpha$-caroteno. $O \beta$-caroteno foi

Tabela 1 - Deslocamentos químicos nos espectros de RMN ${ }^{1} \mathrm{H}$ e RMN ${ }^{13} \mathrm{C}$ da bergenina (1)

\begin{tabular}{|c|c|c|c|}
\hline Posição & $\begin{array}{l}\delta_{H} \\
\text { Experimental } \\
\text { (DMSO, } 400 \mathrm{MHz} \text { ) }\end{array}$ & $\begin{array}{l}\delta_{\mathrm{c}} \\
\text { Experimental } \\
\text { (DMSO, } 100 \mathrm{MHz})\end{array}$ & $\begin{array}{l}\delta_{c} \\
\text { Literatura* } \\
\text { (DMSO, } 75 \mathrm{MHz} \text { ) }\end{array}$ \\
\hline 2 & & 163,6 & 163,4 \\
\hline 3 & & 109,7 & 109,5 \\
\hline 4 & $6,98(s)$ & 151,2 & 151,0 \\
\hline 5 & & 140,8 & 140,6 \\
\hline 6 & & 148,4 & 148,1 \\
\hline 7 & & 116,1 & 116,0 \\
\hline 8 & & 118,3 & 118,1 \\
\hline 9 & $4,98(d, \mathrm{~J}=10,4 \mathrm{~Hz})$ & 72,3 & 72,1 \\
\hline 11 & $\begin{array}{l}3,57(d d d, \mathrm{~J}=9,5,7,6 \mathrm{e} \\
2,0 \mathrm{~Hz})\end{array}$ & 81,9 & 81,8 \\
\hline 12 & $3,20(m)$ & 70,9 & 70,7 \\
\hline 13 & $3,65(t))$ & 73,9 & 73,7 \\
\hline 14 & $\begin{array}{l}3,99(d d, J=10,4 \text { e } 9,5 \\
\mathrm{Hz})\end{array}$ & 80,0 & 79,8 \\
\hline 15 & $3,77(s)$ & 60,1 & 59,9 \\
\hline 16 & $\begin{array}{l}3,85(d d, J=11,7 \text { e } 2,0 \\
H z, 16 a) \\
3,44(m, 16 b)\end{array}$ & 61,3 & 61,1 \\
\hline
\end{tabular}

*Seo et al. (1999). 
majoritário na amostra de uchi apresentando concentração com valores médios de $16,57 \mu \mathrm{g} / \mathrm{g}$. O $\alpha$-caroteno foi observado em traços. Após a separação, o trans- $\beta$-caroteno predominou com $89,3 \%$. Os isômeros 13 -cis- $\beta$-caroteno e 9-cis- $\beta$-caroteno foram detectados, em média $8 \%$ e $3 \%$, respectivamente, confirmando os dados da literatura que indicam esses como os principais isômeros presentes em frutos e legumes (Agostini \& Cecchi, 1996).

Com base na razão de conversão estabelecida pela National Research Council (6 $\mu \mathrm{g}$ de B-caroteno corresponde a 1 $\mathrm{RE}$ - retinol equivalente), verificou-se que $100 \mathrm{~g}$ de polpa de uchi pode contribuir com aproximadamente $27,6 \%$ das necessidades diárias de vitamina A (100 $\mu \mathrm{g} / \mathrm{g} / \mathrm{RE})$ para um homem adulto (NRC,1989).

\section{CONCLUSÃO}

Esse estudo constitui o primeiro registro da presença de bergenina e carotenóides nos frutos de uchi. $\mathrm{O} \beta$-caroteno foi o carotenóide responsável pela maior contribuição para o valor de vitamina A. Considerando a importância do papel nutricional dos carotenóides e a bioatividade do glicosídeo bergenina, esse estudo revelou o potencial desse fruto ocorrente na região amazônica como alimento funcional.

\section{BIBLIOGRAFIA CITADA}

Agostini, T.S.; Cecchi, H.M. 1996. Composição de carotenóides no marolo e em produtos de preparo caseiro. Ciência e Tecnologia de Alimentos, 16(1): 67-71.

Bauerfeind, J.C. 1972. Carotenoid vitamin A precursors and analogs in foods and feeds. Journal of Agricultural and Food Chemistry, 20(3): 456-473.

Chin Y.W.; Kim, J.W. 2006. Three new flavonol glycosides from the aerial parts of Rodgersia podophylla. Chemical \& Pharmaceutical Bulletin, 54(2): 234-236.

Corrêa, M.P. 1984. Diccionário das Plantas Úteis do Brasile das Exóticas Cultivadas. Rio de Janeiro, Imprensa Nacional, V. 6.

Cuatrecasas, J.A. 1961. A taxonomic revision of Humiriaceae, Contribuitions from the United States National Herbarium. Bulletin of the United States National Museum, 35, Part 2.

Davies, B.H. 1976. Chemistry and biochemistry of plants pigments. London: Academic Pres: TW Goodwin, 2.ed, p. 38-165.

Ferreira, C.A.C.; Ramos, J.F.1993. Espécies botânciacs do Campus do INPA: Arbóreas e Arbustivas. Manaus: INPA/OD, Ed. Calderaro.

Harborne J.B,; Baxter, H. 1993. Phytochemical Dictionary. A handbook of Bioactive_Compounds from Plants. Taylor e Francis. London. Washington DC.

Kim, H.S.; Lim, H.K.; Chung, M.W.; Kim, Y.C. 2000. Antihepatotoxic activity of bergenin, the major constituent of Mallotus japonicus, on carbon tetrachloride-intoxicated hepatocytes. Journal of Ethnopharmacology, 69(1): 79-83.
Lim, H.K.; Kim, H.S.; Choi, H.S.; Choi, J.; Kim, S.H.; Chang, M.J. 2001. Effects of bergenin, the major constituent of Mallotus japonicus against D-galactosamine-induced hepatotoxicity in rats. Pharmacology, 63(2): 71-75.

Lim, H.K.; Kim, H.S.; Choi, H.S.; Oh, S.; Choi, J. 2000. Hepatoprotective effects of bergenin, a major constituent of Mallotus japonicus, on carbon tetrachloride-intoxicated rats. Journal of Ethnopharmacology, 72(3): 469-474.

Luna, J.S.; Bento, E.S.; Sant`Ana, A.E.G. 2001. Identificação Estrutural de dois Triterpenóides Pentacíclicos de Endopleura uchi (Humiriaceae); In: 24 ${ }^{\text {a }}$ Reunião Anual da Sociedade Brasileira de Química. Poços de Caldas-MG.

Luna, J.S.; Silva, T.M.; Bento, E.S.; Sant`Ana, A.E. G. 2000. Isolamento e Identificação Estrutural dos Constituintes Químicos de Endopleura uchi (Humiriaceae). In: 23a Reunião Anual da Sociedade Brasileira de Química. Poços de Caldas-MG. http://www.sbq.org.br/ranteriores/23/resumos/0597-1/index. html. Acesso: 09/02/2007.

Ogan, A.U. 1971. Humiriaceae: An isocoumarin from the bark of Sacoglottis gabonensis. Phytochemistry, 10(11): 2832-2833.

Marx, F.; Andrade, E.H.A.; Zoghbi, M.G.B.; Maia, J.G.S. 2002. Studies of edible amazonian plants. Part 5: Chemical characterization of Endopleura uchi fruits. European Food Research and Technology, 214(4): 331-334.

National Research Council (NRC). Recommended dietary allowances. 1989. 10 $10^{\mathrm{a}}$ ed. Washington (DC): National Academy Press.

Rodriguez-Amaya, D.B.; Raymundo, L.C.; Lee, T.C.; Simpson, K.L.; Chichester, C.O. 1976. Carotenoids pigments change in ripening Monordica charantia fruits. Annals of Botany, 40: 615-624.

Seo, E.K.; Chai, H.; Constant, H.L.; Santisuk, T.; Reutrakul, V.; Beecher, C.W.W.; Farnsworth, N.R.; Cordell, G.A.; Pezzuto, J.M.; Kinghorn, A.D. 1999. Resveratrol tetramers from Vatica diospyroides. Journal of Organic Chemistry, 64(19): 6976 -6983.

Shanley, P.; Luz, L.; Swingland, I.R. 2002. The faint promise of a distant market: a survey of Belém's trade in non-timber forest products. Biodiversity and Conservation, 11: 615-636.

Sumino, M.; Sekine, T.; Ruangrungsi, N.; Igarashi, K.; Ikegami, F. 2002. Ardisiphenols and other antioxidant principles from the fruits of Ardisia colorata. Chemical \& Pharmaceutical Bulletin, 50(11):1484-1487.

Taneyana, M.; Yoshida, S.; Kobayash, M.; Hasegawa, M. 1983. Studies on C-glycosides in higher-plants.3. Isolation of norbergenin from Saxifraga stolonifera. Phytochemistry, 22(4): 1053-1054.

Yoshida, T.; Seno, K.; Takama, Y.; Okuda, T. 1982. Bergenin derivatives from Mallotus japonicus. Phytochemistry, 21(5): 1180-1182.

Schultes, R.E. 1979. De plantis toxicariis e mundo novo tropicale commentationes. XXI. Interesting native uses of the Humiriaceae in the northwest Amazon. Journal of Ethnopharmacology, 1: 89-94.

Recebido em 23/07/2007

Aceito em 23/07/2007 